ARTICLE

\title{
Large cation ethylammonium incorporated perovskite for efficient and spectra stable blue light-emitting diodes
}

Zema Chu',2, Yang Zhao1,2, Fei Ma1,2, Cai-Xin Zhang (10 3, Huixiong Deng 2,3, Feng Gao1,2, Qiufeng Ye ${ }^{1,2}$, Junhua Meng ${ }^{1,2}$, Zhigang Yin ${ }^{1,2}$, Xingwang Zhang (i) ${ }^{1,2 凶} \&$ Jingbi You (i) ${ }^{1,2 \otimes}$

Perovskite light-emitting diodes (PeLEDs) have showed significant progress in recent years; the external quantum efficiency (EQE) of electroluminescence in green and red regions has exceeded $20 \%$, but the efficiency in blue lags far behind. Here, a large cation $\mathrm{CH}_{3} \mathrm{CH}_{2} \mathrm{NH}_{2}{ }^{+}$ is added in $\mathrm{PEA}_{2}\left(\mathrm{CsPbBr}_{3}\right)_{2} \mathrm{PbBr}_{4}$ perovskite to decrease the $\mathrm{Pb}-\mathrm{Br}$ orbit coupling and increase the bandgap for blue emission. $\mathrm{X}$-ray diffraction and nuclear magnetic resonance results confirmed that the EA has successfully replaced $\mathrm{Cs}^{+}$cations to form $\mathrm{PEA}_{2}\left(\mathrm{Cs}_{1-x} \mathrm{E} \mathrm{A}_{x} \mathrm{PbBr}_{3}\right)_{2} \mathrm{PbBr}_{4}$. This method modulates the photoluminescence from the green region $(508 \mathrm{~nm})$ into blue $(466 \mathrm{~nm})$, and over $70 \%$ photoluminescence quantum yield in blue is obtained. In addition, the emission spectra is stable under light and thermal stress. With configuration of PeLEDs with $60 \% \mathrm{EABr}$, as high as $12.1 \% \mathrm{EQE}$ of sky-blue electroluminescence located at $488 \mathrm{~nm}$ has been demonstrated, which will pave the way for the full color display for the PeLEDs.

\footnotetext{
${ }^{1}$ Key Laboratory of Semiconductor Materials Science, Institute of Semiconductors, Chinese Academy of Sciences, Beijing 100083, P. R. China. ${ }^{2}$ Center of Materials Science and Optoelectronics Engineering, University of Chinese Academy of Sciences, Beijing 100049, P. R. China. ${ }^{3}$ State Key Lab Superlattices \& Microstruct, Beijing 100083, Institute of Semiconductors, Chinese Academy of Sciences, Beijing 100083, P. R. China. ${ }^{{ }^{凶}}$ email: xwzhang@semi.ac.cn; jyou@semi.ac.cn
} 
ead halide perovskite shows very high potential in display due to its unique emission properties, significant progresses in electroluminescence (EL) for perovskite light-emitting diodes (PeLEDs) have been achieved in recent years ${ }^{1-10}$. The external quantum efficiency (EQE) of red and green emissions have been increased to over than $20 \%^{11-13}$, while the efficiency of blue emission is still lag behind because of the difficulties in synthesizing stable materials and maintaining high quantum efficiency in the films ${ }^{14,15}$.

Several attempts have been done to realize blue emission of the PeLEDs. The easiest way is to incorporate chlorine into brominebased perovskites to tune the bandgap ${ }^{2,4,16-18}$, a 5.7\% EQE in blue from $\mathrm{Br}-\mathrm{Cl}$ halide mixed perovskite has been achieved by Yip et al., recently ${ }^{14}$. However, these $\mathrm{Br}-\mathrm{Cl}$ mixture perovskite could exhibit unavoidable phase separation when exposed to light or under electrical potential ${ }^{4,14,19-21}$, which further induced peak shift or multi-peaks due to the halide migration under applying voltage. The second approach is by modulating the conduction band via metal ions doping such as $\mathrm{Zn}^{2+}, \mathrm{Cu}^{2+}, \mathrm{Mn}^{2+}, \mathrm{Al}^{3+}$ into lead site ${ }^{22-25}$. While the impurity induced by metal elements are considered to be one of the key factors that favors in nonradiative recombination ${ }^{26}$.

To date, the effective attempts to obtain blue PeLEDs are generating the quantum-well structure via reducing-dimensional (quasi-2D and 0D) perovskites with different large cations ${ }^{7,10,17,27-29}$. For example, Sargent et al. have used shorter iso-propylammonium (IPA) molecular to replace long ligands phenylethylammonium (PEA) and tune the quasi-2D $\mathrm{PEA}_{2} \mathrm{~A}_{n-1} \mathrm{~Pb}_{n} \mathrm{X}_{3 n+1}$ perovskite composition with a desired $n$, and showed $1.5 \%$ EQE in sky blue ${ }^{30}$. During preparation of this manuscript, Jin et al. have reported efficient blue light-emitting diodes (LEDs) based on quantum-confined bromide perovskite via using phenylbutylammonium bromide as quasi-2D phases combined with an anti-solvent film deposition method, a peak EQE of up to $9.5 \%$ has been achieved ${ }^{31}$. These strategies are still focus on the modification of the cations in the two dimensional perovskite frame.

In this manuscript, we introduced large cation $\mathrm{CH}_{3} \mathrm{CH}_{2} \mathrm{NH}_{2}{ }^{+}$ (EA) into the Cs ${ }^{+}$site in $\mathrm{PEA}_{2}\left(\mathrm{CsPbBr}_{3}\right)_{2} \mathrm{PbBr}_{4}$ perovskite, and tuned the emission from green $(508 \mathrm{~nm})$ into blue $(466 \mathrm{~nm})$. The obtained perovskite films showed larger than $70 \%$ photoluminescence quantum yield (PLQY) in blue, and more importantly, the blue emission shows good spectra stability under lightsoaking and heating. Eventually, by optimizing the content of EABr, a $12.1 \%$ EQE of the sky-blue $(488 \mathrm{~nm})$ EL was obtained, which could be the highest efficiency for blue emission PeLEDs so far.

\section{Results}

The thin films characterization. To obtain high efficient blue PeLEDs, quasi-2D perovskite $\mathrm{PEA}_{2}\left(\mathrm{CsPbBr}_{3}\right)_{n-1} \mathrm{PbBr}_{4}(n=3$, see "Methods") with efficient green emission as the initial composition for modification. The emission wavelength of quasi-2D $\mathrm{PEA}_{2}(\mathrm{CsPbBr})_{2} \mathrm{PbBr}_{4}$ was located at $508 \mathrm{~nm}$ as shown in Fig. $1 \mathrm{a}$. The three-dimensional perovskite $\mathrm{CsPbBr}_{3}$ was chosen as the main core of the emission, excess of $\mathrm{CsBr}\left(\mathrm{CsBr}: \mathrm{PbBr}_{2}=2: 1\right.$, molar ratio) has been introduced to passivate the non-radiative defects recombination ${ }^{9}$. The long alkyl chain phenylethylammonium bromide (PEABr) has been generally introduced in the perovskites in order to form a $2 \mathrm{D}$ phases when it replaces A-site atoms of the outer, which has been demonstrated to confine the charge and enhance emission ${ }^{10}$.

The quasi-2D $\left.\mathrm{PEA}_{2}(\mathrm{CsPbBr})_{2}\right)_{2} \mathrm{PbBr}_{4}$ perovskite films with different ratio of $\mathrm{EABr}$ were synthesized through mixing $\mathrm{PbBr}_{2}$, $\mathrm{CsBr}, \mathrm{PEABr}$, and $\mathrm{EABr}$ in the solution of dimethyl sulfoxide
(DMSO) and a one-step spin-coating process (details in "Methods"). As shown in Fig. 1a, the emission of films can be tuned from $508 \mathrm{~nm}$ to $466 \mathrm{~nm}$ with increasing EA cations from 0 to $100 \%$. The emission images of these samples under ultraviolet excitation $(365 \mathrm{~nm})$ are shown in Supplementary Fig. 1, consistent with PL results, the band edge absorption also showed a blue-shift (Fig. 1b). The PLQY has been increased from $42 \%$ for the control film to around $70 \%$ while less than $60 \%$ is introduced, it was also found that too much $\mathrm{EABr}(\geq 80 \%)$ lead to the decrease of PLQY (Fig. 1d). The increasing PLQY could be due to the suppression of non-radiative recombination by EABr doping. The reduction of PLQY with the increasing EA cation could be explained that amount of EA has not been introduced into $\mathrm{CsPbBr}$, resulting non-radiative recombination centers. The details in emission properties of these samples are plotted in Supplementary Fig. 2 and summarized in Supplementary Table 1.

The spectra stability is a critical issue for blue emission of perovskite materials. The photo and thermal stability of quasi-2D perovskite film with $60 \% \mathrm{EABr}$ sealed with $40 \mathrm{mg} \mathrm{ml}^{-1}$ poly (methyl methacrylate) (PMMA) as example were studied. The PL spectra of the quasi-2D perovskite with EABr was kept under continuous ultraviolet light illumination $\left(360 \mathrm{~nm}, 1 \mathrm{~mW} \mathrm{~cm}^{-2}\right)$ for $240 \mathrm{~min}$ (Fig. 1d, Supplementary Fig. 3a). The blue perovskite film also illustrates good thermal stability, both of the PL peak position and FWHM were not changed after annealing at $60^{\circ} \mathrm{C}$ for $270 \mathrm{~min}$ (Fig. 1e, Supplementary Fig. 3b). In comparison, we found the $\mathrm{Cl}-\mathrm{Br}$ mixed perovskite $\mathrm{PEA}_{2} \mathrm{CsPb}\left(\mathrm{Br}_{0.1} \mathrm{Cl}_{0.9}\right)_{3} \mathrm{PbBr}_{4}$ with emission owns blue region exhibited poor spectral stability (Supplementary Fig. 4).

We carried out the $\mathrm{X}$-ray diffraction (XRD) characterization for $\mathrm{PEA}_{2}\left(\mathrm{EA}_{x} \mathrm{Cs}_{1-x} \mathrm{PbBr}_{3}\right)_{2} \mathrm{PbBr}_{4}$ with different $\mathrm{EABr}$ to check the phases (Fig. 2b). The XRD patterns of the thin film without $\mathrm{EABr}$ shows three main peaks at $15.42^{\circ}, 30.37^{\circ}$, and $31.03^{\circ}$, which match well with the (100) and (200) planes of $\mathrm{CsPbr}_{3}$ and (006) of $\mathrm{Cs}_{4} \mathrm{PbBr}_{6}$ respectively. It is found that the diffraction peaks from (100) of $\mathrm{CsPbBr}_{3}$ and (006) of $\mathrm{Cs}_{4} \mathrm{PbBr}_{6}$ become weaker and finally disappear with increasing EA cation. More importantly, the main diffraction at $30.37^{\circ}$ gradually decreased to $30.26^{\circ}$, due to the large molecular size of EA cation $\left(\mathrm{EA}^{+}, 274 \mathrm{ppm}\right)$ compared with cesium cation $\left(\mathrm{Cs}^{+}, 177 \mathrm{ppm}\right)^{32}$, we can anticipate that the EA cation has been filled into the crystal lattice of $\mathrm{CsPbBr}_{3}$ to form $\mathrm{Cs}_{1-x} \mathrm{EA}_{x} \mathrm{PbBr}_{3}(0 \leq x \leq 1)$ perovskite phase in quasi-2D structure (Fig. 2a). To confirm the successful incorporation of $\mathrm{EABr}$ in the final quasi-2D perovskite films, we measured the ${ }^{1} \mathrm{H}$ NMR spectra of the EABr, PEABr powder and the quasi-2D perovskite films with or without $\mathrm{EABr}$, which were dissolved in DMSO- $d_{6}$. The EA signals from EABr power were completely keeping in line with the quasi-2D perovskite with EABr film, as shown in Fig. 2d, which manifested the EA cation can been filled into the quasi-2D perovskite.

To further confirm the insertion of EA in $\mathrm{CsPbBr}_{3}$ crystal lattice, the XRD patterns of pure 3D perovskite of $\mathrm{CsPbr}_{3}$ with different EABr were collected (Fig. 2c). Similarly, the introduction of $\mathrm{EABr}$ lead to the decrease of the diffraction angles, indicating an expansion of the $\mathrm{CsPbBr}_{3}$ crystal lattice by insertion of the larger EA cations. The emission wavelength and optical bandgap of these perovskite films can be tuned from green to blue region by adding EABr into $\mathrm{CsPBr}_{3}$ (Supplementary Fig. 5). It might be argued that a possibility the EA leads to formation of the quasi$2 \mathrm{D}$ perovskites by acting as a capping ligand. In that case, we should observe emission or absorption features from two dimensional perovskites in mixed perovskites. However, while the samples with moderate amount of $\mathrm{EABr}(x<0.75)$ show only one dominant emission peak and no excitonic absorption peaks at higher energy, providing evidence that these films are only simple phases. Whiling introducing more $\operatorname{EABr}(x \geq 0.75)$ in 



Fig. 1 Characteristics of perovskite films with different EABr. a Normalized steady photoluminescence (PL) spectra. b UV-vis absorption spectra, and c Photoluminescence quantum yield (PLQY) spectra. d Normalized PL spectra of quasi-2D perovskite film with $60 \%$ EABr under continuous UV radiation $\left(360 \mathrm{~nm}, 1 \mathrm{~mW} \mathrm{~cm}^{-2}\right)$ for different exposure times. e Normalized PL spectra of quasi-2D perovskite film with $60 \%$ EABr after continuous thermal treatment $\left(60^{\circ} \mathrm{C}\right)$ for different times.

$\mathrm{CsPbBr}$, the films exhibit two photoluminescence peaks and an excitonic absorption, the higher energy emission and sharp absorption peaks is almost overlap with $\mathrm{EAPbBr}_{3}$ itself, indicating that EA maybe not completely go into the $\mathrm{CsPbBr}_{3}$ while the EA amount is too much. These results are consistent with the results shown in Fig. 1c and could also explain why the PLQY of the $\mathrm{PEA}_{2}(\mathrm{CsPbBr})_{2} \mathrm{PbBr}_{4}$ is low while incorporating too much EABr. As we know, the size of guanidinium (GA) molecular is similar as the cation of EA molecular ${ }^{32}$. The GA molecular have been tried to tune the emission of quasi-two dimensional perovskites, unfortunately, it is found that the GA molecular cannot tune the emission from green region into blue (Supplementary Fig. 6).

We carried out morphologies characterization of the perovskite films with different EABr by atomic force microscopy (AFM) and scanning electron microscopy (SEM) (Supplementary Figs. 7 and 8 ). The root mean square roughness of these films are around $0.5 \mathrm{~nm}$, the smooth film will avoid leakage current. In addition, the crystal size has not been decreased while introducing of the EA into the $\mathrm{PEA}_{2}\left(\mathrm{Cs} \mathrm{PbBr}_{3}\right)_{2} \mathrm{PbBr}_{4}$ perovskite, indicating that the blue shift of the emission could not be originated from quantum confinement effect.

Density functional theory (DFT) calculation. To gain further insights regarding the origin of the band gap increasing in the quasi-2D perovskite, we carried on theoretical band structure calculations within DFT using $\mathrm{CsPbBr}_{3}, \mathrm{Cs}_{0.75} \mathrm{EA}_{0.25} \mathrm{PbBr}_{3}$ and $\mathrm{Cs}_{0.5} \mathrm{EA}_{0.5} \mathrm{PbBr}_{3}$ as the model system. The details are provided in the "Methods" section. A $4 \times 3 \times 4 \mathrm{k}$-mesh for orthorhombic unit cell $\mathrm{CsPbBr}_{3}$ is performed in relaxation (Fig. 3a). All atomic coordinates and the lattice parameters are relaxed until forces of each atom were less than $0.02 \mathrm{eV}$. The hybrid functional exchange-correlation functional of Heyd-Scuseria-Ernzerhof (HSE06) $^{33}$ with 25\% Hartree-Fock is considered at accurate calculation of electronic structures. The band structure of $\mathrm{CsPbBr}$ is shown in Fig. 2b. The calculated electronic density of states (DOS) of $\mathrm{CsPbBr}_{3}, \mathrm{Cs}_{0.75} \mathrm{EA}_{0.25} \mathrm{PbBr}_{3}$, and $\mathrm{Cs}_{0.5} \mathrm{EA}_{0.5} \mathrm{PbBr}_{3}$ is shown in Fig. $3 c$, the calculated corresponding bandgaps are 2.30, 2.58 , and $2.60 \mathrm{eV}$, which are in good agreement with the experimental observations in the absorption spectra $(2.34,2.37$, and $2.41 \mathrm{eV}$, Supplementary Fig. 5b).

The first-principle calculation of three-dimensional perovskite has confirmed the increasing of band gap with introduction of EA cation into $\mathrm{Cs} \mathrm{PbBr}_{3}$ lattice. It might be argued that the increase of lattice will generally lead to decrease the bandgap, such as $\mathrm{MAPbBr}_{3}$ and $\mathrm{FAPbBr}_{3}$ compared with $\mathrm{CsPbBr}$, and why the large size of EA insertion lead to the enlargement of bandgap of $\mathrm{CsPbBr} 3$ ? It could be simply explained that the uneven lattice expansion makes the length of the six $\mathrm{Pb}-\mathrm{Br}$ bonds increase differently, and resulting different decreases for valence band maximum (VBM) and conduction band minimum $(\mathrm{CBM})$ in energy. As we know, the bandgap of the $\mathrm{APbBr}_{3}$ is determined by $\mathrm{Pb}-\mathrm{Br}$ orbitals coupling ${ }^{34}$, the longest bonding $\mathrm{Pb}-\mathrm{Br}$ length with the smallest coupling will determine the CBM, while the shortest $\mathrm{Pb}-\mathrm{Br}$ bond with the strongest coupling will control the VBM. The insertion of EA cation could mainly affect the shortest $\mathrm{Pb}-\mathrm{Br}$ 
a


Fig. 2 Crystal composition of perovskite films. a Schematics showing the EA cation doping in the perovskite lattice to replace Cs ${ }^{+}$in quasi-2D perovskite. b X-ray diffraction (XRD) pattern of the quasi-2D perovskite with different ratio of EABr. The inset is amplified XRD pattern of samples from $29^{\circ}$ to $32^{\circ}$. c XRD patterns of the $\mathrm{Cs}_{1_{-x}} \mathrm{EA}_{x} \mathrm{PbBr}_{3}(0 \leq x \leq 1)$ perovskite thin films. d ${ }^{1} \mathrm{H}$ NMR spectra of the $\mathrm{EABr}, \mathrm{PEABr}$ powers and quasi-2D perovskite with or without $\mathrm{EABr}$.

bond which lower the VBM of obviously, and increase the bandgap. More fundamental study of the $\mathrm{Cs}_{1-x} \mathrm{EA}_{x} \mathrm{PbBr}_{3}$ system will be carried out to explore the clear reason of the band gap enlargement via EA introduction in the near future. The ultraviolet photoelectron spectroscopy (UPS) results showed that the enlargement of bandgap after introducing EA is mainly due to the downshift of the VBM (Fig. 3d, Supplementary Figs. 9a, b), further confirming the insertion of EA into the $\mathrm{CsPbBr}_{3}$ lattice. 
a

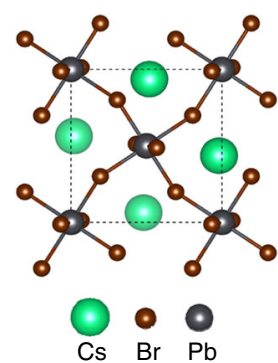

C

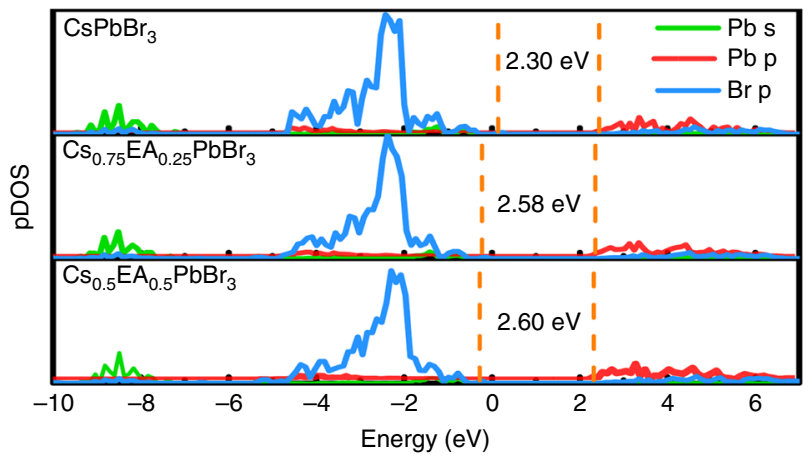

d

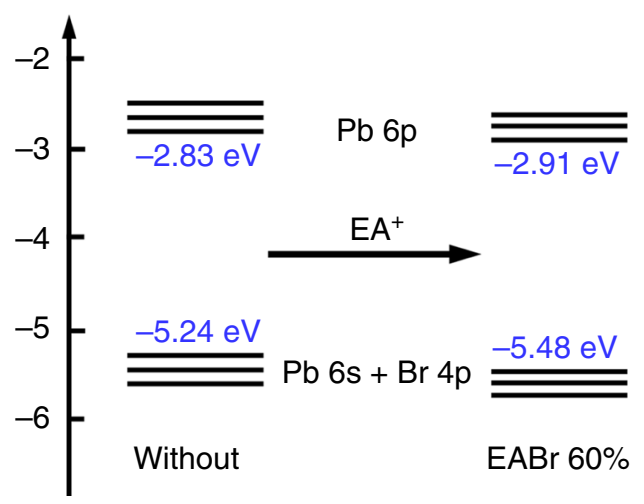

Fig. 3 The crystal structures and electronic structures of different perovskites. a The crystal structures of $\mathrm{CsPbBr}_{3}$. b The electronic structures of $\mathrm{CsPbBr}_{3}$. c The calculated electronic density of states (DOS) of $\mathrm{CsPbBr}_{3}, \mathrm{Cs}_{0.75} \mathrm{EA}_{0.25} \mathrm{PbBr}_{3}$, and $\mathrm{Cs}_{0.5} \mathrm{EA}_{0.5} \mathrm{PbBr}_{3}$. d Schematic representation of variation of energy levels of quasi-2D perovskite in $\mathrm{Pb} 6 \mathrm{p}$ and $(\mathrm{Pb} 6 \mathrm{~s}+\mathrm{Br} 4 \mathrm{p})$ orbitals on insertion of EA cation. The valence band maximum (VBM) and conduction band minimum ( $C B M)$ come from $(\mathrm{Pb} 6 \mathrm{~s}+\mathrm{Br} 4 \mathrm{p})$ and $\mathrm{Pb} 6 \mathrm{p}$ orbitals, respectively.

\section{Discussion}

Encouraged by above findings, we constructed LEDs using with $\mathrm{PEA}_{2}\left(\mathrm{Cs}_{1-x} \mathrm{EA}_{x} \mathrm{PbBr}_{3}\right)_{2} \mathrm{PbBr}_{4}$ as active layer. A device structure glass/ITO/m-PEDOT:PSS $(35 \mathrm{~nm}) /$ Perovskite $(25 \mathrm{~nm}) /$ TBPi $(40 \mathrm{~nm}) / \mathrm{LiF}(1 \mathrm{~nm}) / \mathrm{Al}(70 \mathrm{~nm})$ is adopted, in which m-PEDOT:PSS is PSS-Na modified PEDOT:PSS to increase the work function forming better level alignment for hole injection and electron blocking ${ }^{10,35}$. The schematic of the band alignment diagram and the cross-section SEM image of the completed device are shown in Supplementary Fig. 10.

The performance of the PeLEDs are characterized and summarized in Fig. $4 \mathrm{a}-\mathrm{c}$ and Table 1 . It can be found that the maximum brightness are 2790, 2191, and $83 \mathrm{~cd} \mathrm{~m}^{-2}$ (Fig. 4a), the EQE are $13.3 \%, 12.1 \%$ and $4.19 \%$ (Fig. $4 \mathrm{~b}$ ) and the EL are located at 495,488 and $480 \mathrm{~nm}$ (Fig. $4 \mathrm{c}$ ) for $40 \%, 60 \%$ and $80 \% \mathrm{EABr}$, respectively. The normalized EL spectra of PeLEDs with different ratios of $\mathrm{EABr}$ was obtained at $6 \mathrm{~V}$ and all the emissions held up narrow full-widths at half-maximum (FWHMs) of $\leq 25 \mathrm{~nm}$, resulting in the high color purity in Commission Internationale de l'Eclairage (CIE) coordinate as shown in Fig. 4e. It is obvious that the EL image of varying EABr was in line with the result of EL spectra and CIE (Fig. 4f). With the blue shift of EL spectra, the corresponding CIE value and the EL image of different EABr also changed from the green to blue region, indicating the successful achievement of a blue-emissive perovskite LED. To our best knowledge, the $12.1 \%$ EQE for sky-blue emission at $488 \mathrm{~nm}$ could be the best for the PeLEDs. The lower efficiency while shifting the emission into deeper blue region could be due to the low PLQY and also large poor injection (Figs. 1c and 4a). A histogram of peak EQEs for 28 devices shows an average value of $\sim 9.5 \%$ in Supplementary Fig. 11, the statistical data mean that the performance of PeLEDs device owns good reproducibility.

The spectra stability of EL under applied voltage was tested, it is clear that the normalized EL spectra of PeLEDs with $60 \% \mathrm{EABr}$ is unchanged with the increasing applied voltage (Fig. 4d), suggesting that the emission spectra is stable under applied voltage. We have collected about 12 min video of our LEDs working at a constant current density of $0.3 \mathrm{~mA} \mathrm{~cm}^{-2}$, it can be found that EL show the constant color at varying the time (Supplementary Movie 1). In comparison, as similar as photoluminescence, the EL spectra changed for mixed halide perovskite with increasing the voltage (Supplementary Fig. 12). We have carried out the device stability test at a constant current density of $1.5 \mathrm{~mA} \mathrm{~cm}^{-2}$ and also at the $100 \mathrm{~cd} \mathrm{~m}^{-2}$, we found that the device without encapsulation could survive around $1 \mathrm{~h}$ in nitrogen glove box (Supplementary Fig. 13). The increasing input voltage at constant current brings increasing input power, suggesting that increasing Joule heating takes place in the device, which could contribute for instability of PeLEDs.

In conclusion, we have demonstrated that the blue emission of perovskite LEDs with EA cation incorporation into threedimensional perovskite lattice. The emission has been tuned from green into blue, and showed high PLQY $(>70 \%)$. In addition, the emission spectra is stable under photo and thermal stress. With tailoring the EA composition, an efficient PeLEDs with 12.1\% EQE in $488 \mathrm{~nm}$ emission has been achieved. This concept is expected to open an avenue for full color display using PeLEDs.

\section{Methods}

Materials. $\mathrm{CsBr}, \mathrm{PbBr}_{2}$, DMSO and poly (sodium 4-styrenesulfonate) (PSS-Na, average $\mathrm{Mw} \sim 70,000)$ were purchased from Sigma-Aldrich. 2,2',2"-(1,3,5-Benzinetriyl)-tris(1-phenyl-1-H-benzimidalzole) (TBPi), and ethylam-momium bromide $(\mathrm{EABr})$ were purchased from Xi'an Polymer Technology Corp. PEABr was purchased from Dyesol (now Greatcell Solar). The modified PEDOT:PSS (m-PEDOT: PSS) solution is mix of normal PEDOT:PSS (AI 4083) aqueous solution and $100 \mathrm{mg} \mathrm{ml}^{-1}$ PSS-Na aqueous solution by a volume ration of $3: 1$.

Perovskite solution preparation. The $\mathrm{Cs}_{x} \mathrm{EA}_{1-x} \mathrm{PbBr}_{3}$ and $\mathrm{PEA}_{2} \mathrm{PbBr}_{4}$ precursor solution were precursor solution were prepared by dissolving appropriately stoichiometric $\mathrm{CsBr}, \mathrm{EABr}, \mathrm{PEABr}$, and $\mathrm{PbBr}_{2}$ in $\mathrm{DMSO}$ under continuous stirring for $4 \mathrm{~h}$ at room temperature, keeping the molar concentration $\mathrm{PbBr}_{2}$ at $0.1 \mathrm{M}$. The $\mathrm{CsPbBr}_{3}$ precursor solution was also prepared by dissolving $\mathrm{CsBr}$ and $\mathrm{PbBr}_{2}(2: 1$ in molar) in DMSO under continuous stirring for $4 \mathrm{~h}$ at room temperature, keeping $\mathrm{Pb}^{2+}$ at $0.1 \mathrm{M}$, too. The quasi-2D perovskite $\mathrm{PEA}_{2}\left(\mathrm{Cs} \mathrm{PbBr}_{3}\right)_{2} \mathrm{PbBr}_{4}$ precursor solution was mixed the $\mathrm{PEA}_{2} \mathrm{PbBr}_{4}$ and $\mathrm{CsPbBr}_{3}$ by a volume ration of $1: 2$. The ratio of $\mathrm{m} \% \mathrm{EABr}$ refers to the molar between $\mathrm{EABr}$ and $\mathrm{PbBr}_{2}$.

Device fabrication. The indium tin oxide (ITO)-coated glass substrates were sequentially cleaned by sonication in detergent, deionized water, acetone and isopropyl alcohol and then dried by $\mathrm{N}_{2}$. After a ultraviolet ozone treatment for ITO in $10 \mathrm{~min}$, the modified PEDOT:PSS aqueous solution was spin-coated onto the ITO substrate at $9000 \mathrm{rpm}$ for $60 \mathrm{~s}$ and baked at $160^{\circ} \mathrm{C}$ for $15 \mathrm{~min}$ in ambient air. Thereafter, the substrates were transferred into a nitrogen-filled glove box, and the 

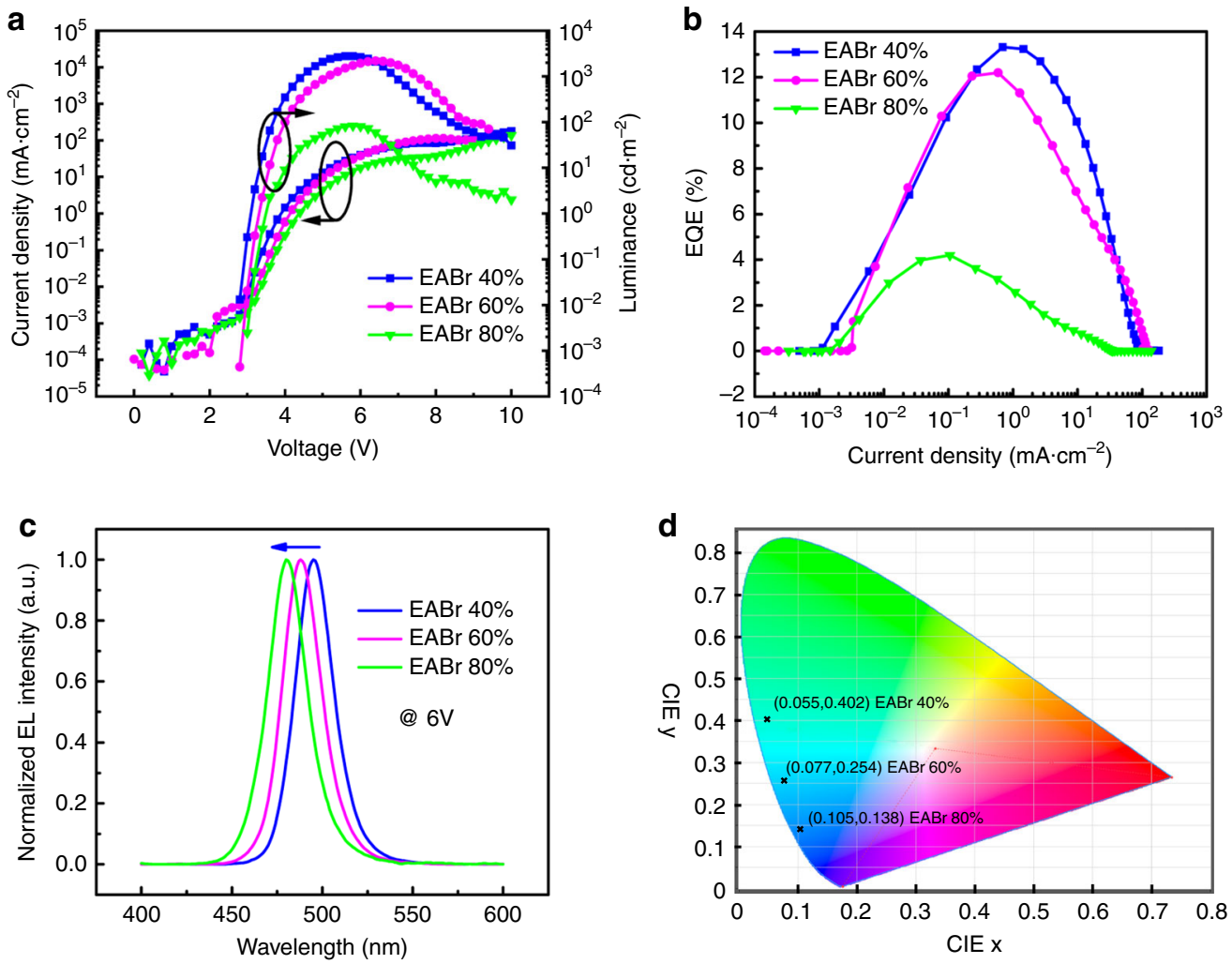

e

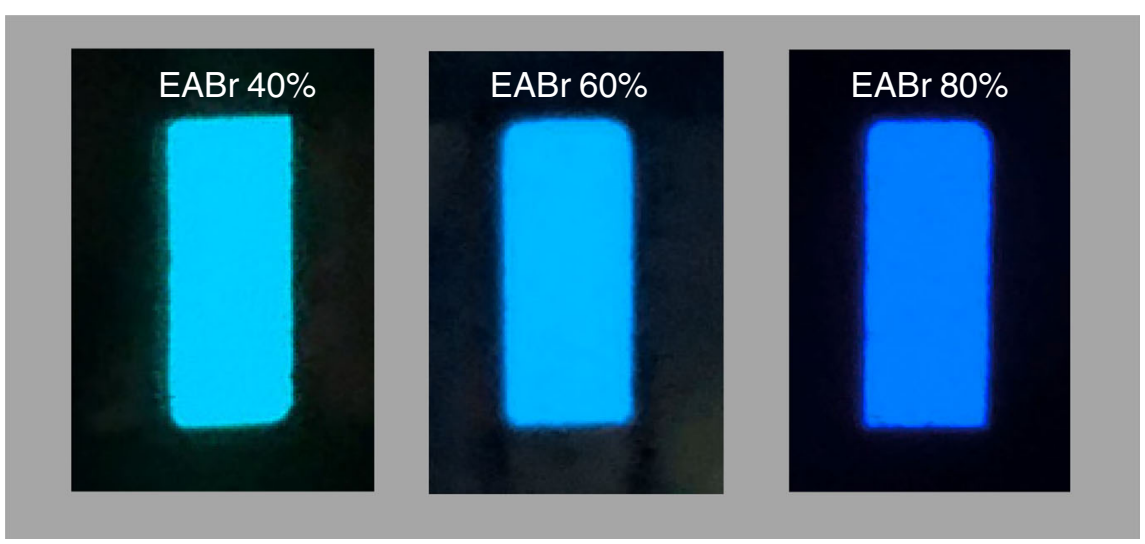

Fig. 4 Performance of the PeLEDs with different ratio of EABr. a Characterization of current density and luminance versus applied voltage.

b Characterization of external quantum efficiency (EQE) versus current density. c Normalized electroluminescence (EL) spectra of perovskite LEDs with $60 \% \mathrm{EABr}$ under increasing applied voltage. d Commission Internationale de I'Eclairage (CIE) values of the EL spectra of perovskite LEDs. e Electroluminescence images of the perovskite LEDs with different EABr.

\section{Table 1 Summary of quasi-2D perovskite LEDs with different ratios of EABr.}

\begin{tabular}{lllllll} 
EABr ratio & Max. EQE (\%) & Max. CE (cd.A-1) & Max. L. (cd.m $\left.\mathbf{- n}^{-2}\right)$ & EL peak (nm) & FWHM (nm) & CIE \\
\hline $40 \%$ & 13.3 & 24.45 & 2790 & 495 & 23 & $(0.055,0.402)$ \\
$60 \%$ & 12.1 & 17.58 & 2191 & 488 & 25 & $(0.077,0.254)$ \\
$80 \%$ & 4.19 & 6.17 & 83 & 480 & 25 & $(0.105,0.138)$
\end{tabular}

LED light-emitting diode, EABr ethylammonium bromide, EQE external quantum efficiency, CE current efficiency, L. luminance, EL electroluminescence, FWHM full-width at half-maximum, CIE Commission Internationale de I'Eclairage.

perovskite precursor solution was then spin-coated on the m-PEDOT:PSS film at $3000 \mathrm{rpm}$ for $2 \mathrm{~min}$, followed by annealing on a hot plate at $60^{\circ} \mathrm{C}$ for $20 \mathrm{~min}$. Finally, the fabrication of PeLEDs was completed by depositing TBPi $(40 \mathrm{~nm})$ and $\mathrm{LiF} / \mathrm{Al}$ electrodes $(1 \mathrm{~nm} / 100 \mathrm{~nm})$ layer by layer through a shadow mask in high vacuum thermal evaporator. The device area was $10 \mathrm{~mm}^{2}$ as defined by overlapping area of ITO and Al electrode.
First-principle calculations. The electronic structures of $\mathrm{CsPbBr}_{3}$, $\mathrm{Cs}_{0.75} \mathrm{EA}_{0.25} \mathrm{PbBr}_{3}$, and $\mathrm{Cs}_{0.5} \mathrm{EA}_{0.5} \mathrm{PbBr}_{3}$ are calculated using DFT methods (employing the ab initio code VASP) ${ }^{36}$ with the projector-augmented wave (PAW) pseudopotentials ${ }^{37}$. A plane wave cutoff energy of $400 \mathrm{eV}$ and the Perdew-BurkeErnzerhof revised for solids (PBEsol) exchange-correlation functional are employed $^{38}$. 
Perovskite film and device characterizations. The SEM images were obtained using a field-emission SEM (FEI NanoSEM650), which used an electron beam accelerated at $500 \mathrm{~V}$ to $30 \mathrm{kV}$, enabling operation at a variety of currents. AFM measurements were carried out using a Bruker FASTSCANBIO in non-contact mode. The XRD patterns of the perovskite thin films were performed with a Rigaku D/max $2500 \mathrm{H}$ equipment with a conventional $\mathrm{Cu}$ target $\mathrm{X}$-ray tube (Cu K-alpha, $\lambda=1.5405$ $\AA$ ) as the X-ray source. Scans were taken with a $0.5-\mathrm{mm}$-wide source and detector slits, with X-ray generator settings at $40 \mathrm{kV}$ and $30 \mathrm{~mA}$. Proton nuclear magnetic resonance $\left({ }^{1} \mathrm{H}\right.$ NMR) spectra were recorded on Bruker Avance II $400 \mathrm{MHz}$ system with BBI probe. Steady-state PL spectra of the perovskite films was measured at room temperature in the ambient air using a FLS1000 spectrometer. Absorption spectra were acquired using an ultraviolet-visible spectrometer (Cary 5000). PLQYs of the perovskite thin films were recorded by a commercialized PLQY measurement system (LQE-50-PL) from Enlitech with excitation from a $368 \mathrm{~nm}$ LED. UPS spectra was performed on a Thermo Scientific ESCALab250Xi with an applied bias of $-10 \mathrm{~V}$. The He I emission line at $21.22 \mathrm{eV}$ was employed. The Helium pressure in the analysis chamber during measurement was about $3 \times 10^{-8}$ mbar. The film samples over the ITO layer had a conductive connection with an Au sample, so the Fermi level value of the film samples is equal to that of Au samples. The work function $\Phi$ (that is the Fermi level absolute value of the free film) of the test films can be calculated from following equation: $h v-\Phi=E_{\mathrm{Fermi}}-E_{\text {cutoff, where }} E_{\mathrm{Fermi}}$ and $E_{\text {cutoff }}$ is the value of Fermi level position and the steep edge position in UPS spectrum, respectively, $h v=21.22 \mathrm{eV}$ and $E_{\mathrm{Fermi}}=21.51 \mathrm{eV}$. The I-V-L curve, EL spectrum, EQE, CIE, and operating lifetime of the perovskite LED were carried out simultaneously by a commercialized system (LQE-50-EL, Enlitech) that was equipped with an integrated sphere and photomultiplier tubes (PTM), in which the PMT is used to measure the low luminance. All the device characterization tests of perovskite LEDs were recorded at room temperature in the ambient air for the un-encapsulated devices, except that the operational stability test was carried out in an $\mathrm{N}_{2}$-filled glovebox. To confirm our measurement results, cross-checking with other research groups was carried out. We tried our best to encapsulate the devices and measured at the different research units, and found that the measurement results are almost consistent. Some of the devices showed about $10 \%$ difference, which could be due to the degradation during the transmission.

\section{Data availability}

The data that support the findings of this study are available from the corresponding author upon request.

Received: 18 November 2019; Accepted: 25 July 2020;

Published online: 20 August 2020

\section{References}

1. Tan, Z.-K. et al. Bright light-emitting diodes based on organometal halide perovskite. Nat. Nanotechnol. 9, 687-692 (2014).

2. Song, J. et al. Quantum dot light-emitting diodes based on inorganic perovskite cesium lead halides $\left(\mathrm{CsPbX}_{3}\right)$. Adv. Mater. 27, 7101-7108 (2015).

3. Cho, H. et al. Overcoming the electroluminescence efficiency limitations of perovskite light-emitting diodes. Science 350, 1222-1225 (2015).

4. Li, G. et al. Highly efficient perovskite nanocrystal light-emitting diodes enabled by universal crosslinking method. Adv. Mater. 28, 3528-3534 (2016).

5. Yuan, M. et al. Perovskite energy funnels for efficient light-emitting diodes. Nat. Nanotechnol. 11, 872-877 (2016).

6. Wang., N. et al. Perovskite light-emitting diodes based on solution-processed self-organized multiple quantum wells. Nat. Photonics 10, 699-704 (2016).

7. Chen, Z. et al. High-performance color-tunable perovskite light emitting devices through structural from bulk to layered film. Adv. Mater. 29, 1603157 (2017).

8. Xiao, Z. et al. Efficient perovskite light-emitting diodes featuring nanometersized crystallites. Nat. Photonics 11, 108-115 (2017).

9. Zhang, L. et al. Ultra-bright and highly efficient inorganic based perovskite light-emitting diodes. Nat. Commun. 8, 15640-15648 (2017).

10. Yang, X. et al. Efficient green light-emitting diodes based on quasi-two dimensional composition and phase engineered perovskite with surface passivation. Nat. Commun. 9, 570-578 (2018).

11. Lin, K. et al. Perovskite light-emitting diodes with external quantum efficiency exceeding 20 percent. Nature 562, 245-248 (2018).

12. Cao, Y. et al. Perovskite lighting-emitting diodes based on spontaneously formed submicrometre-scale structures. Nature 562, 249-253 (2018).

13. Chiba, T. et al. Anion-exchange red perovskite quantum dots with ammonium iodine salts for highly efficient light-emitting devices. Nat. Photonics 12, 681-687 (2018).

14. Li, Z. et al. Modulation of recombination zone position for quasi-twodimensional blue perovskite light-emitting diodes with efficiency exceeding 5\%. Nat. Commun. 10, 1027 (2019).

15. Vashishtha, P., Ng, M., Shivarudraiah, S. B. \& Halpert, J. E. High efficiency blue and green light-emitting diodes using Ruddlesdenpopper inorganic mixed halide perovskites with butylammonium interlayers. Chem. Mater. 31, 83-89 (2018)

16. Yao, E. P. et al. High-brightness blue and white LEDs based on inorganic perovskite nanocrystals and their composites. Adv. Mater. 29, 1606895 (2017)

17. Gangishetty, M. K., Hou, S., Quan, Q. \& Congreve, D. N. Reducing architecture limitations for efficient blue perovskite light-emitting diodes. $A d v$ Mater. 30, 1706226 (2018).

18. Pan, J. et al. Highly efficient perovskite-quantum-dot light-emitting diodes by surface engineering. Adv. Mater. 28, 8718-8725 (2016).

19. Tang, X. et al. Local observation of phase segregation in mixed-halide perovskite. Nano Lett. 18, 2172-2178 (2018).

20. Vashishtha, P. \& Halpert, J. E. Field-driven ion migration and color instability in red-emitting mixed halide perovskite nanocrystal light-emitting diodes. Chem. Mater. 29, 5965-5973 (2017)

21. Draguta, S. et al. Rationalizing the light-induced phase separation of mixed halide organic-inorganic perovskites. Nat. Commun. 8, 200 (2017).

22. Ward van der, S. et al. Highly emissive divalent-ion-doped colloidal CsPb1$\mathrm{xMxBr3}$ perovskite nanocrystals through cation exchange. J. Am. Chem. Soc. 139, 4087-4097 (2017).

23. Bi, C. et al. Thermally stable copper(II)-doped cesium lead halide perovskite quantum dots with strong blue emission. J. Phys. Chem. Lett. 10, 943-952 (2019).

24. Hou, S., Gangishetty, M. K., Quan, Q. \& Congreve, D. N. Efficient blue and white perovskite light-emitting diodes via manganese doping. Joule 2, 2421-2433 (2018)

25. Liu, M. et al. Aluminum-doped cesium lead bromide perovskite nanocrystals with stable blue photoluminescence used for display backlight. Adv. Sci. 4, 1700335 (2017).

26. Liu, W. et al. Mn2+-doped lead halide perovskite nanocrystals with dual-color emission controlled by halide content. J. Am. Chem. Soc. 138, 14954-14961 (2016).

27. Congreve, D. N. et al. Tunable light-emitting diodes utilizing quantumconfined layered perovskite emitters. ACS Photonics 4, 476-481 (2017).

28. Wang, Q., Ren, J., Peng, X. F., Ji, X. X. \& Yang, X. H. Efficient sky-blue perovskite light-emitting devices based on ethylammonium bromide induced layered perovskites. ACS Appl. Mater. Int. 9, 29901-29906 (2017).

29. Kumar, S. et al. Efficient blue electroluminescence using quantum-confined two-dimensional perovskites. ACS Nano 10, 9720-9729 (2016).

30. Xing, J. et al. Color-stable highly luminescent sky-blue perovskite lightemitting diodes. Nat. Commun. 9, 3541 (2018).

31. Liu, Y. et al. Efficient blue light-emitting diodes based on quantum-confined bromide perovskite nanostructures. Nat. Photonics 13, 760-764 (2019).

32. Gholopour, S. et al. Globularity-selected large molecules for a new generation of multication perovskites. Adv. Mater. 29, 1702005 (2017).

33. Krukau, A. V. et al. Influence of the exchange screening parameter on the performance of screened hybrid functionals. J. Chem. Phys. 125, 224106 (2006).

34. Mittal, M. et al. Size of the organic cation tunes the band gap of colloidal organolead bromide perovskite nanocrystals. J. Phys. Chem. Lett. 7, 3270-3277 (2016).

35. Zou, C. T. \& Ding, L. M. Modified PEDOT layer makes a $1.52 \mathrm{~V}$ VOC for perovskite/PCBM solar cells. Adv. Energy Mater. 7, 1601193 (2017).

36. Kresse, G. \& Furthermuller, J. Effect of Er doping on the electronic structure optical properties of ZnO. Phys. Rev. B: Condens. Matter Mater. Phys. 54, 11169-11186 (1996).

37. Kresse, G. \& Joubert, D. From ultrasoft pseudopotentials to the projector augmented-wave method. Phys. Rev. B 59, 1758-1775 (1999).

38. Csonka, G. I. et al. Assessing the performance of recent density functionals for bulk solids. Phys. Rev. B 79, 155107 (2009).

\section{Acknowledgements}

This work is supported by National Key Research and Development Program of China (Grant No. 2018YFB0406700), National Natural Science Foundation of China (Grant Numbers: 61925405, 61634001, 11874347 and 61922077) and Strategic Priority Research Program of the Chinese Academy of Sciences (XDB43000000) The authors would like to thanks Prof. Jianpu Wang from Nanjing Tech University in China for kind helping in Angular distribution of radiation intensity measurement.

\section{Author contributions}

Z.C. and J.Y. conceived the idea, J.Y. directed and supervised the project. Z.C. fabricated and characterized the devices, Y.Z., F.M., Q.Y., J.M., F.G., Z.Y., and X.Z. were involved in data analysis. C.Z. and H.D. conducted theoretical calculations. Z.C., X.Z., and J.Y. cowrote the manuscript. All authors contributed to discussions and finalizing the manuscript.

\section{Competing interests}

The authors declare no competing interests. 


\section{Additional information}

Supplementary information is available for this paper at https://doi.org/10.1038/s41467020-17943-6.

Correspondence and requests for materials should be addressed to X.Z. or J.Y.

Peer review information Nature Communications thanks Yizheng Jin, Weijun Ke and the other, anonymous, reviewer(s) for their contribution to the peer review of this work.

Reprints and permission information is available at http://www.nature.com/reprints

Publisher's note Springer Nature remains neutral with regard to jurisdictional claims in published maps and institutional affiliations. (c) (P) Open Access This article is licensed under a Creative Commons Attribution 4.0 International License, which permits use, sharing, adaptation, distribution and reproduction in any medium or format, as long as you give appropriate credit to the original author(s) and the source, provide a link to the Creative Commons license, and indicate if changes were made. The images or other third party material in this article are included in the article's Creative Commons license, unless indicated otherwise in a credit line to the material. If material is not included in the article's Creative Commons license and your intended use is not permitted by statutory regulation or exceeds the permitted use, you will need to obtain permission directly from the copyright holder. To view a copy of this license, visit http://creativecommons.org/ licenses/by/4.0/.

(C) The Author(s) 2020 